\title{
Effects of Dufour and Soret on Unsteady MHD Heat and Mass Transfer Flow Past a Semi-Infinite Moving Vertical Plate in a Porous Medium with Viscous Dissipation
}

\author{
M. N. RajaShekar
}

\begin{abstract}
This paper investigates the Dufour and Soret effects on unsteady MHD free convection flow past a semi-infinite moving vertical plate in a porous medium considering the viscous dissipation effects. The resulting governing equations are transformed into non linear ordinary differential equations using similarity transformation. The set of non linear ordinary differential equations are first linearized by using Quasi-linearization technique and then solved numerically by using implicit finite difference scheme. Then the system of algebraic equations is solved by using Gauss-Seidal iterative method. The solution is found to be mainly dependent on six governing parameters including the magnetic field parameter $\mathrm{M}$, the suction parameter v0, Soret number $\mathrm{Sr}$, Dufour number Du, Eckert number Ec, and Darcian parameter Da. Numerical results are tabulated for the local Nusselt number and Sherwood number. Velocity, Temperature and Concentration profiles drawn for different controlling parameters reveal the tendency of the solution.
\end{abstract}

Index Terms-Magnetic field effects, Soret and Dufour effects, suction, viscous dissipation.

\section{INTRODUCTION}

Combined heat and mass transfer (or double-diffusion) in fluid-saturated porous media finds applications in a variety of engineering processes such as heat exchanger devices, petroleum reservoirs, chemical catalytic reactors and processes, geothermal and geophysical engineering moisture migration in a fibrous insulation and nuclear waste disposal and others. Double diffusive flow is driven by buoyancy due to temperature and concentration gradients. Bejan and Khair [1] investigated the free convection boundary layer flow in a porous medium owing to combined heat and mass transfer.

Magneto hydrodynamic flows have applications in meteorology, solar physics, cosmic fluid dynamics, astrophysics, geophysics and in the motion of earth's core. In addition from the technological point of view, MHD free convection flows have significant applications in the field of stellar and planetary magnetospheres, aeronautical plasma flows, chemical engineering and electronics.

Raptis [2] studied mathematically the case of time varying two dimensional natural convective flow of an incompressible, electrically conducting fluid along an infinite vertical porous plate embedded in a porous medium. Elabashbeshy [3] studied heat and mass transfer along a vertical plate in the presence of magnetic field. Chamkha and
Khaled [4] investigated the problem of coupled heat and mass transfer by magneto hydrodynamic free convection from an inclined plate in the presence of internal heat generation or absorption.

In the combined heat and mass transfer processes, it is known that the thermal energy flux resulting from concentration gradients is referred to as the Dufour or diffusion-thermal effect. Similarly, the Soretor thermo-diffusion effect is the contribution to the mass fluxes due to temperature gradients. The Dufour and Soret effects may be significant in the areas of geosciences and chemical engineering. Kafoussias and Williams [5] employed the finite difference method to examine the Dufour and Soret effects on mixed free-forced convective heat and mass transfer along a vertical surface, various other influences that have been considered include magnetic field [6], variable suction [7] and chemical reaction [8].

Various other aspects dealing with the Soret effect on the combined heat and mass transfer problems have been also studied. For example, Joly et al. [9] used the brinkman-extended Darcy model to examine the effect of the soret effect on the onset of convective instability. Mojtabi et al. [10] presented, making use of as pseudo-spectral chebyshev collocation method, a stability analysis of the influence of vibration on Soret-driven convection in porous media. Bourich et al. [11] carried out an analytical and numerical study of the onset of soret convection in a horizontal porous layer subjected to a uniform vertical magnetic field.

However in the existing convective heat transfer literature on the non-Newtonian fluids, the effect of the viscous dissipation has been generally disregarded. Gnaneswara and Bhasker Reddy [12] have studied the effects of soret and dufour on steady MHD free convection flow in a porous medium with viscous dissipation. Kishan and Shashidar Reddy [13] have studied the MHD effects on non-Newtonian power-law fluid past a continuously moving porous flat plate with heat flux and Viscous Dissipation. Recently Alamet al. [14] has studied Dufour and Soret effects on unsteady MHD free convection and Mass transfer flow past a vertical porous plate in a porous medium. The present work aims to study the effects of Dufour and Soret on unsteady free convection and mass transfer flow past an infinite vertical porous flat plate in porous medium by taking into account the effect of viscous dissipation.

\section{Mathematical ANALYsis}

M. N. Raja Shekar is with the JNTUH College of Engineering Jagityal, Nachupally, Karimnagar-505 501, Andhra Pradesh, India (e-mail: mnrs71@ gmail.com).
An unsteady two-dimensional flow of an incompressible 
and electrically conducting viscous fluid, along an infinite vertical porous flat plate embedded in a porous medium is considered. The $\mathrm{x}$-axis is taken along the infinite plate, and parallel to the free-stream velocity which is vertical and the $\mathrm{y}$-axis is taken normal to the plate. A magnetic field $\mathrm{B}_{0}$ of uniform strength is applied transversely to the direction of the flow.

Initially the plate and the fluid are at same temperature $\mathrm{T}_{\infty}$ in a stationary condition with concentration level $\mathrm{C}_{\infty}$ at all points. For $t>0$, the plate starts moving impulsively in its own plane with a velocity $U_{0}$, its

Temperature is raised to $T_{\omega}$ and the concentration level at the plate is raised to $C_{\omega}$. The fluid is assumed to have constant properties except that the influence of the density variations with temperature and concentration, which are considered only in the body force term. Under the above assumptions, the physical variables are functions of $y$ and $t$ only. Assuming that the Boussinesq and boundary-layer approximation hold and using the Darcy-Forchheimer model, the basic equations, which govern the problem, are given by Alam and Rahman [8]:

$$
\begin{gathered}
\frac{\partial v}{\partial y}=0 \\
\frac{\partial u}{\partial t}+v \frac{\partial u}{\partial y}=\boldsymbol{v} \frac{\partial^{2} u}{\partial y^{2}}+g \beta\left(T-T_{\infty}\right)+g \beta^{*}\left(C-C_{\infty}\right)-\frac{\sigma B_{0}^{2} u}{\rho}- \\
\frac{v}{K} u-\frac{b}{K} u^{2} \\
\frac{\partial T}{\partial t}+v \frac{\partial T}{\partial y}=\alpha \frac{\partial^{2} T}{\partial y^{2}}+\frac{D_{m} K_{T}}{c_{S} c_{p}} \frac{\partial^{2} C}{\partial y^{2}}+\frac{v}{C p}\left(\frac{\partial u}{\partial y}\right)^{2} \\
\frac{\partial C}{\partial t}+v \frac{\partial C}{\partial y}=D_{M} \frac{\partial^{2} C}{\partial y^{2}}+\frac{D_{m} K_{T}}{T_{m}} \frac{\partial^{2} T}{\partial y^{2}}
\end{gathered}
$$

Initially $(\mathrm{t}=0)$ the fluid and the plate are at rest. Thus the no slip boundary conditions at the surface of the plate for the above problem for $\mathrm{t}>0$ are:

$$
\begin{gathered}
u=U_{0}, \quad v=v(t), \quad T=T_{w}, \quad C=C_{w} \text { at } \quad y=0 \\
u=0, \quad T=T_{\infty}, \quad C=C_{\infty}, \text { as } y \rightarrow \infty
\end{gathered}
$$

The last but one term on the right-hand side of the energy equation (3) and concentration equation (4) signifies the Dufour or diffusion-thermo effect and the Soretor thermal-diffusion effect, respectively.

Here $\mathrm{u}, \mathrm{v}$ are the Darcian velocity components in the $\mathrm{x}$ - and $\mathrm{y}$-directions respectively, $\mathrm{t}$ is the time, $\boldsymbol{v}$ is the kinematic viscosity, $g$ is the acceleration due to gravity, $\rho$ is the density, $\beta$ is the coefficient of volume expansion, $\beta^{*}$ is the volumetric coefficient of expansion with concentration, $\mathrm{k}$ is Darcy permeability, $b$ is the empirical constant, $\mathrm{B}_{0}$ is magnetic induction, $\mathrm{T}$ and $\mathrm{T}_{\infty}$ are the temperature of the fluid inside the thermal boundary layer and the fluid temperature in the free stream, respectively, while $\mathrm{C}$ and $\mathrm{C}_{\infty}$ are the corresponding concentrations. Also,$\sigma$ is the electric conductivity, $\alpha$ is the thermal diffusivity, $D_{m}$ is the coefficient of mass diffusivity, $c_{p}$ is the specific heat at constant pressure, $T_{m}$ is the mean fluid temperature, $K_{T}$ is the thermal diffusion ratio and $c_{\mathrm{s}}$ is the concentration susceptibility.

\section{Method of SOlution}

Now in order to obtain a local similarity solution (in time) of the problem under consideration, we introduce a time dependent length scale $\delta$ as

$$
\delta=\delta(t)
$$

In terms of this length scale, a convenient solution of the equation (1) is considered to be in the following form:

$$
V=v(t)=-v_{0} \frac{\boldsymbol{v}}{\boldsymbol{\delta}}
$$

where $v_{0}>0$ is the suction parameter.

We now introduce the following dimensionless variables:

$$
\begin{gathered}
\eta=\frac{y}{\delta}, \\
u=u_{0} f(\eta), \\
\theta(\eta)=\frac{T-T_{\infty}}{T_{w}-T_{\infty}}, \\
\varphi(\eta)=\frac{C-C_{\infty}}{C_{w}-C_{\infty}},
\end{gathered}
$$

Then introducing the relations (6)-(8) into the equations (2)-(3) respectively, we obtain (by using the analysis of Sattar and Hossain [9] and Hasimoto [10]), the following dimensionless ordinary differential equations:

$$
\begin{gathered}
f^{\prime \prime}+\left(2 \eta+v_{0}\right) f^{\prime}+G_{r} \theta+G_{m} \varphi-M f-\frac{1}{D_{a}} f- \\
\frac{R_{e} F_{s}}{D_{a}} f^{2}=0 \\
\theta^{\prime \prime}+p_{r}\left(2 \eta+v_{0}\right) \theta^{\prime}+p_{r} D_{u} \varphi^{\prime \prime}+p_{r} E_{c}\left(f^{\prime \prime}\right)^{2}=0 \\
\varphi^{\prime \prime}+S_{c}\left(2 \eta+v_{0}\right) \varphi^{\prime}+S_{c} S_{r} \theta^{\prime \prime}=0
\end{gathered}
$$

where primes denotes differentiation with respect to $\eta$ and the dimensionless quantities are given by $D a=\frac{k}{\delta^{2}}$ is the local Darcy number, $F_{\mathrm{s}}=\frac{b}{\delta}$ is local Forchheimer number, $R_{e}=\frac{U_{0} \delta}{v}$ is the local Reynolds number, $P_{r}=\frac{v}{\alpha}$ is the Prandtl number, $S_{c}=\frac{v}{D_{m}}$ is the Schmidt number, $M=\frac{\sigma B_{0}^{2} \delta^{2}}{v \rho}$ is the magnetic field parameter, $S_{r}=\frac{D_{m} K_{T}\left(T_{w}-T_{\infty}\right)}{T_{m} v\left(C_{w}-C_{\infty}\right)}$ is the Soret number, $D=\frac{D_{m} K_{T}\left(C_{w}-C_{\infty}\right)}{c_{s} c_{p} v\left(T_{w}-T_{\infty}\right)}$ is the Dofour number, $G_{r}=\frac{g \beta\left(T_{w}-T_{\infty}\right) \delta^{2}}{v U_{0}}$ is the local Grashof number and $G_{m}=\frac{g \beta^{*}\left(C_{w}-C_{\infty}\right) \delta^{2}}{v U_{0}}$ is the local modified Grashof number.

The corresponding boundary conditions for $\mathrm{t}>0$ are obtained as

$$
\begin{aligned}
& f=1, \theta=1, \quad \varphi=1, \quad \text { at } \eta=0 \\
& f=0, \theta=0, \quad \varphi=0, \quad \text { as } \eta \rightarrow \infty
\end{aligned}
$$

The equations (9)-(11) are locally similar in time but not explicitly time dependent. 
To solve the system of transformed governing equations (9) where

$\&$ (11) with the boundary conditions (12), we first linearized equation (9) by using Quasi linearization technique.

Then equation (9) is transformed to

$$
\begin{array}{r}
f^{\prime \prime}+\left(2 \eta+v_{0}\right) f^{\prime}+G_{r} \theta+G_{m} \varphi-M f-\frac{1}{D_{a}} f- \\
\frac{R_{e} F_{s}}{D_{a}}\left(2 F f-F^{2}\right)=0(13)
\end{array}
$$

and

$$
\begin{aligned}
& D_{0}[i]=2 C_{0}[i]+h C_{1}[i], D_{1}[i]=-4 C_{0}[i], \\
& D_{2}[i]=2 C_{0}[i]-h C_{1}[i], D_{3}[i]=2 h^{2} C_{2}[i]
\end{aligned}
$$

$$
H_{0}[i] \varphi[i+1]+H_{1}[i] \varphi[i]+H_{2}[i] \varphi[i-1]+H_{3}[i]=0
$$

where

$$
\begin{aligned}
& H_{0}[i]=2 E_{0}[i]+h E_{1}[i], H_{1}[i]=-4 E_{0}[i] \\
& H_{2}[i]=2 E_{0}[i]-h E_{1}[i], H 3[i]=2 h^{2} E_{2}[i]
\end{aligned}
$$

$$
A_{0} f^{\prime \prime}+A_{1} f^{\prime}+A_{2} f+A_{3}=0
$$

where

$$
\begin{gathered}
A_{0}[i]=1, \quad A_{1}[i]=2 \eta+\gamma_{0}, \\
A_{2}[i]=-m-\frac{1}{D a}-\frac{2 \operatorname{Re} F_{s}}{D a} F \\
A_{3}[i]=-\frac{\operatorname{Re} F_{s}}{D a} F^{2}+G_{r} \theta+G_{m} \phi
\end{gathered}
$$

where ' $h$ ' represents the mesh size in $\eta$ direction. Equation (17), (18) and (19) are solved under the boundary conditions (12) by Thomas algorithm for various parameters entering into the problem and computations were carried out by using $\mathrm{C}$ programming.

Knowing the concentration field, the rate of mass transfer coefficient can be obtained which in the non-dimensional form in terms of Sherwood number is given by

$$
S_{h}=-\left(\frac{\partial C}{\partial y}\right)_{y=0}
$$

Equation (10) can be expressed as

$$
C_{0} \theta^{\prime \prime}+C_{1} \theta^{\prime}+C_{2}=0
$$

where

$$
\begin{aligned}
& C_{0}[i]=1, \quad C_{1}[i]=P_{r}\left(2 \eta+\gamma_{0}\right) \\
& C_{2}[i]=P_{r} D \phi^{\prime \prime}+P_{r} E c\left(F^{\prime \prime}\right)^{2}
\end{aligned}
$$

And Equation (11) can be expressed as

$$
E_{0} \phi^{\prime \prime}+E_{1} \phi^{\prime}+E_{2}=0
$$

where

$$
E_{0}[i]=1 E_{1}[i]=S_{C}\left(2 \eta+\gamma_{0}\right), E_{2}[i]=S_{C} S_{r} \theta^{\prime \prime}
$$

Using implicit finite difference formulae, the equations (14), (15) \& (16) are transformed to

$$
B_{0}[i] f[i+1]+B_{1}[i] f[i]+B_{2}[i] f[i-1]+B_{3}[i]=0
$$

where

$$
\begin{gathered}
B_{0}[i]=2 A_{0}[i]+h A_{1}[i], \\
B_{1}[i]=-4 A_{0}[i]-h A_{1}[i]+2 h^{2} A_{2}[i], B_{2}[i]=2 A_{0}[i], \\
B_{3}[i]=2 h^{2} A_{3}[i] . \\
D_{0}[i] \theta[i+1]+D_{1}[i] \theta[i]+D_{2}[i] \theta[i-1]+D_{3}[i]=0
\end{gathered}
$$

Knowing the temperature field, the rate of heat coefficient can be obtained which in the non-dimensional form in terms of the Nusselt number is given by $N_{u}=-\left(\frac{\partial \theta}{\partial y}\right)_{y=0}$

\section{RESUlTS AND DisCUSSIONS}

A Parametric study is performed to explore the effect of suction parameter, on the velocity, Soret and Dufour numbers temperature and concentration profiles. The numerical computations have been done for different value of $V_{0}, S_{r}, D$ and for fixed values of $P_{r}, S_{c}, G_{r}, G_{m}, R_{e}$ and $F_{\mathrm{s}}$. The values of $S_{\mathrm{r}}$ and $D$ are taken in such a way that their product should be a constant provided the mean temperature is also constant. The value of Prandtlnumber is chosen as 0.71 which corresponds to air and the value of Schmidt number is chosen to represent hydrogen at $25^{\circ} \mathrm{c}$ and $1 \mathrm{~atm}$. The values of Grash of numbers are taken to represent the free convection problem as $G r=12$ and $G m=6$. The value of local Reynolds number is taken as $R e=100$ and local For chheimernumber is chosen as $F_{\mathrm{s}}=$ 0.09 .

TABLE I: THE VALUES OF NUSSELT NUMBER AND SHERWOOD NUMBER

FOR DIFFERENT VALUES OF SR AND DU
\begin{tabular}{|l|l|l|l|}
\hline $\mathrm{S}_{\mathrm{r}}$ & $\mathrm{D}_{\mathrm{u}}$ & $\mathrm{N}_{\mathrm{u}}$ & $\mathrm{S}_{\mathrm{h}}$ \\
\hline 2.0 & 0.03 & 1.121243 & 1.102232 \\
\hline 1.0 & 0.06 & 1.009123 & 1.312547 \\
\hline 0.5 & 0.12 & 0.921737 & 1.810015 \\
\hline 0.4 & 0.15 & 0.885147 & 1.904895 \\
\hline 0.2 & 0.30 & 0.863917 & 1.942347 \\
\hline
\end{tabular}

The values of Nusselt number and the Sherwood number which are directly proportional to $-\theta^{\prime}(0)$ and $-\emptyset^{\prime}(0)$ are calculated and presented in the above tabular form for various values of $S_{r}, D_{u}$. It is evident from the table that as $S_{r}$ 
decreases there is an increase in Sherwood number but decrease in Nusselt number.

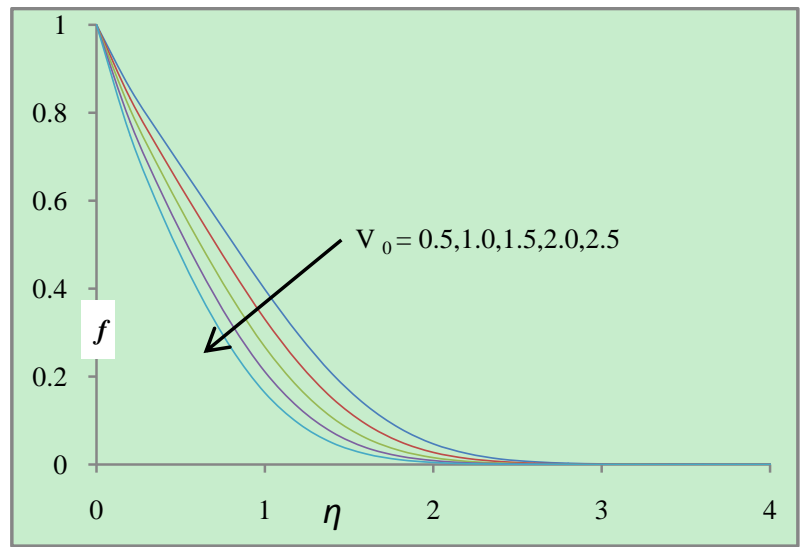

Fig. 1. Velocity profile for different values of v for $S r=2.0, D=0.03, D a$ $=0.5$ and $M=0.3$.

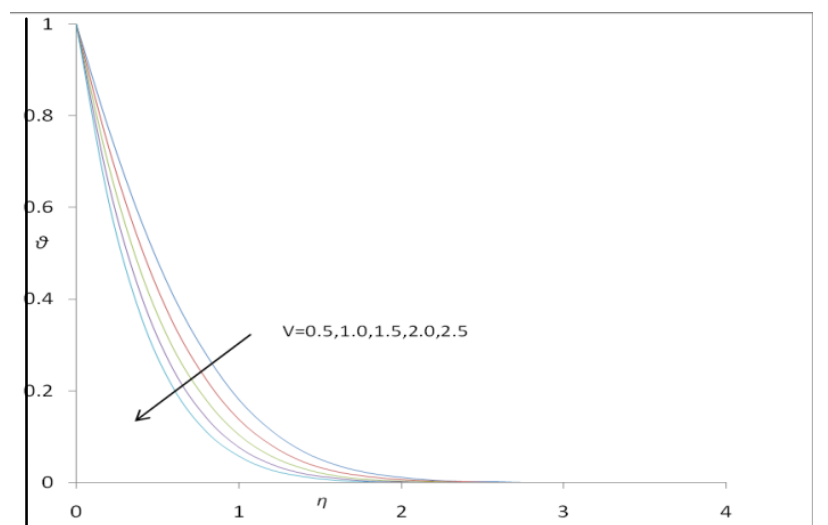

Fig. 2. Temperature Profile for different values of $v$ for $S r=2.0, D=0.03, D a$ $=0.5$ and $M=0.3$.

The effect of suction parameter on the velocity profiles in shown in Fig. 1. It is noticed from the figure that increase in the suction parameter decreases the velocity which indicates that suction stabilizes the growth in the boundary layer. Fig. 2 shows the effect of suction parameter on the temperature profiles, from which it is evident that the temperature decreases as there is an increase in suction parameter.

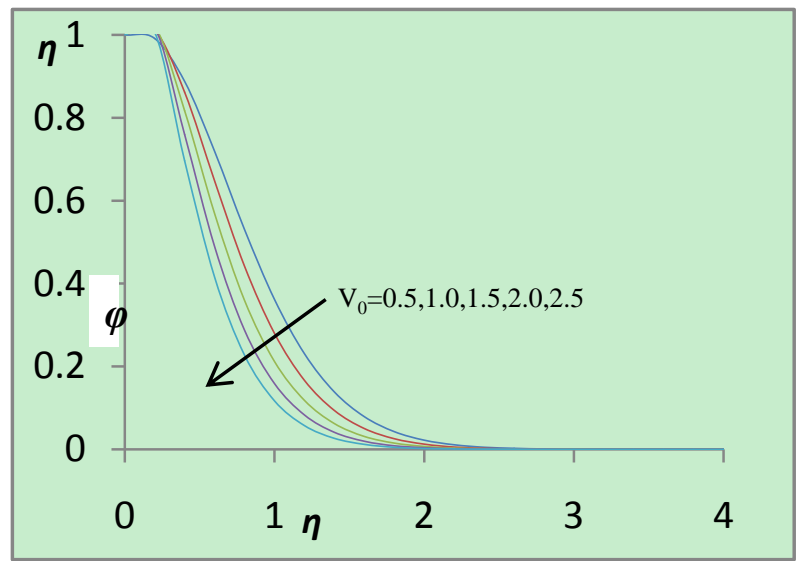

Fig. 3. Concentration Profile for different values of v for $S r=2.0, D=0.03$, $D a=0.5$ and $M=0.3$.

The influence of suction parameter on concentration profiles is displayed i Fig. 3. It is observed from the figure that the concentration decreases with an increase in suction parameter away from the wall where as a reverse phenomenon is seen near the wall, i.e. the concentration increases with the increase in suction parameter very near to the wall. Hence suction reduces the growth of the thermal and concentration boundary layers.

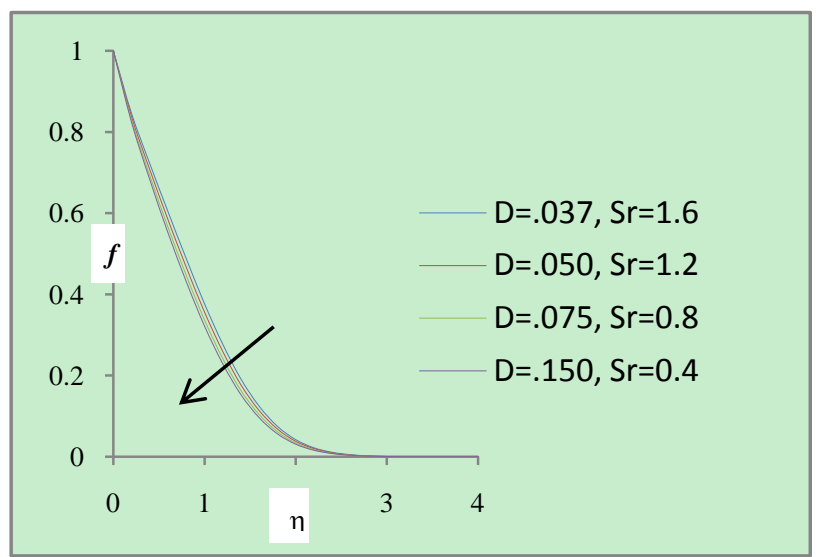

Fig. 4. Velocity Profile for different values of $D, S r$ for $v=0.5, D a=0.5$ and $M=0.3$.

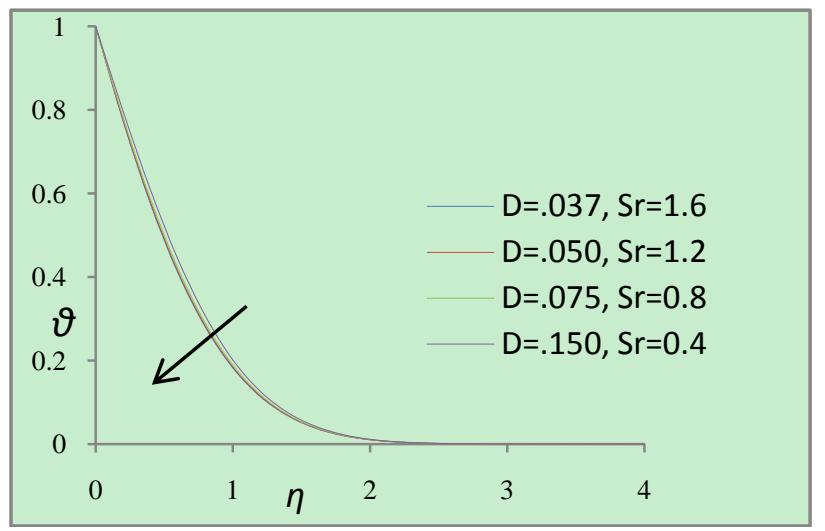

Fig. 5. Temperature Profile for different values of $D, S r$ for $V 0=0.5, D a$ $=0.5$ and $M=0.3$.

The effects of Soret and Dufou rnumbers on velocity profiles and temperature profiles are shown in Fig. 4 and Fig. 5 respectively. As there is a decrease in the Soret number or an increase in the Dufour number, the velocity and temperature decreases. Here the variation in the profiles is very low.

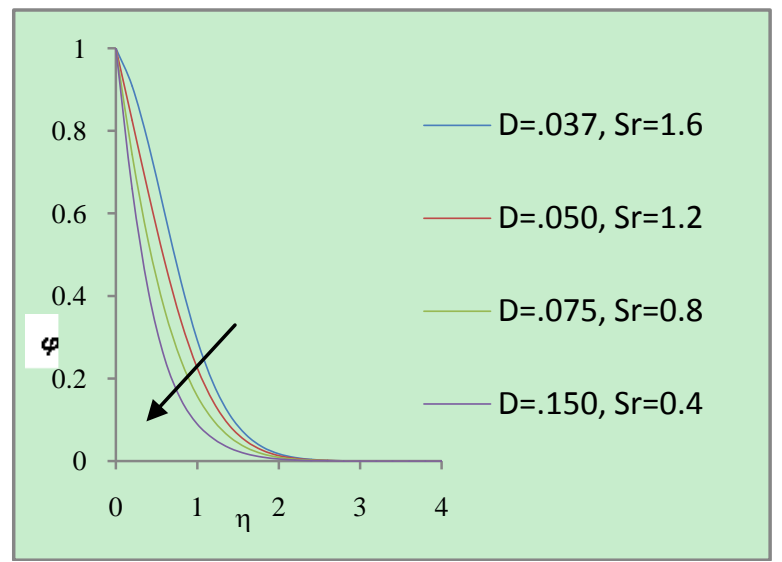

Fig. 6. Concentration Profile for different values of $D, S r$ for $v=0.5, D a=0.5$ and $M=0.3$.

The variation in concentration profiles with the change in soret and dufour number is displayed in Fig. 6. The concentration increases as there is an increase in Dufournumber or decrease in Soretnumber. The viscous dissipation effect is to decelerate the temperature profiles. 


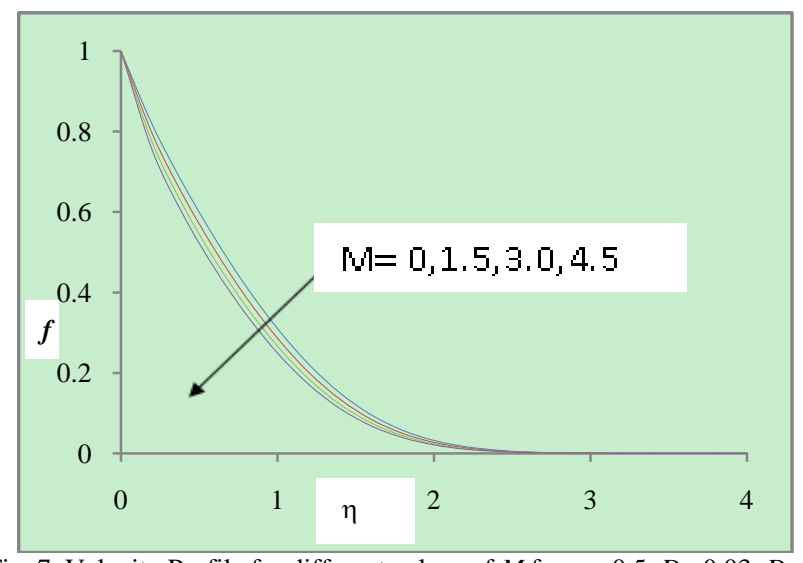

Fig. 7. Velocity Profile for different values of $M$ for $v=0.5, D=0.03, D a=$ 0.5 and $S r=2.0$.

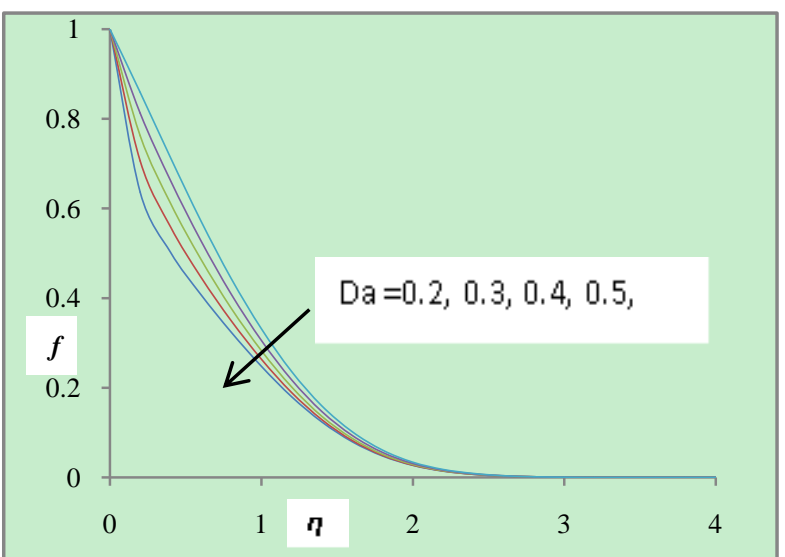

Fig. 8. Velocity Profile for different values of $D a$ for $v=0.5, M=0.3, D=0.03$ and $S r=2.0$.

The effect of magnetic field on the velocity profiles is shown in Fig. 7. It is evident from the figure that the increase in the magnetic field parameter decreases the velocity profiles. The effect of magnetic field is to decrease the velocity. The variation in velocity profiles with the change in Darcy parameter is shown in figure 8 . It is noticed from the figure that the velocity increases with the increase in Darcy parameter.

\section{CONCLUSIONS}

1) Suction reduces the growth of the hydrodynamic, thermal and concentration boundary layers.

2) The increase in the Dufour number or decrease in the Soret number decreases the velocity and temperature profiles whereas increases the concentration.

3) Viscous Dissipation decelerates the temperature.

4) Magnetic field retards the motion of the fluid whereas Darcy number accelerates the velocity.

\section{REFERENCES}

[1] A. Bejan and K. R. Khair, "Heat and mass transfer by natural convection in a porous medium," Int. J. Heat Mass Transfer, vol. 28, pp. 909-918, 1985.

[2] A. Raptis, "Flow through a porous medium in the presence of magnetic field," Int. J.Energy Res., vol. 10, pp. 97-101,1986.

[3] E. M. A. Elabashbeshy, "Heat and mass transfer along a vertical plate with variable temperature and concentration in the presence of magnetic field," Int. J. Eng. Sci., vol. 34, pp. 515-522, 1997.

[4] A. J. Chamkha and A. R. A. Khaled, "Similarity solutions for hydromagnetic simultaneous heat and mass transfer by natural convection froman inclined plate with internal heat generation or absorption," Heat Mass Transfer, vol. 37, pp.117-123,2001.

[5] N. G. Kafoussias and E. W. Williams, "Thermal-diffusion and diffusion-thermo effects on mixed free-forced convective and mass transfer boundary layer flow with temperature dependent viscosity," International Journal of Engineering Science, vol. 33, no. 9, pp. 1369-1384, 1995.

[6] A. Postelnicu, "Influence of a magnetic field on heat and mass transfer by natural convection from vertical surfaces in porous media considering Soret and Dofour effects," International journal of Heat and Mass Transfer, vol. 47, pp. 1467-1472, 2004.

[7] M. S. Alam and M. M. Rahman, "Dofour and Soret effects on mixed convection flow past a vertical porous flat plate with variable suction," Nonlinear Analysis: Modelling and control, vol. 11, no. 1, pp. 3-12, 2006.

[8] A. Postelnicu, "Influence of chemical reaction on heat and mass transfer by natural convection from vertical surfaces in porous media considering Soret and Dufour effects," Heat Mass Transfer, vol. 43, pp. 595-602, 2007.

[9] F. Joly, P. Vasseur and G. Labrosse, "Soret instability in a vertical Brinkman porous enclosure," Numerical Heat Transfer. Part A: Applications, vol. 39, no. 4, pp. 339-359, 2001.

[10] M. C. C. Mojtabi, Y. P. Razi, K. Maliwa, and A. Mojtabi, "Influence of Soret-driven convection in porous media," Numerical Heat Transfer. Part A: Applications, vol. 46, no. 10, pp. 981-993, 2004.

[11] M. Bourich, M. Hasnaoui, A. Amahmid, M. Er-Raki and M. Mamou, "Analytical and numerical study of combined effects of a magnetic field and an external shear stress on Soret convection in a horizontal porous enclosure," Heat Transfer. Part A: Applications, vol. 54, no. 11, pp. 1042-1060, 2008.

[12] M. G. Reddy and N. B. Reddy, "Soret and Dufour effects on steady MHD free convection flow past a semi-infinite moving vertical plate in a porous medium with viscous dissipation," Int. J. of Appl. Math and Mech., vol. 6, no. 1, pp. 1-12, 2010.

[13] N. Kishan and B. S. Reddy, "MHD effects on non-Newtonian power-law fluid past a continuously moving porous flat plate with heat flux and viscous dissipation," International journal of Applied mechanics and engineering, vol. 1, no. 4, pp. 425- 445, 2012.

[14] M. S. Alam, M. M. Rahman, and M. A. Samad, "Dufour and Soret effects on unsteady MHD free convection and mass transfer flow past a vertical porous plate in a porous medium," Nonlinear Analysis modeling and control, vol. 11, no. 3, pp. 217-226, 2006

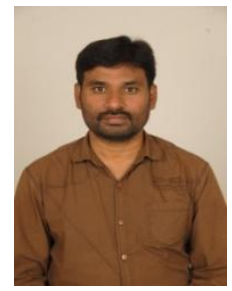

M. N. Raja Shekar has obtained his Ph.D. degree in 2000 in the area of the Fluid dynamics. He has completed his M. Sc. (Applied Mathematics) and B. Sc. from the prestigious Osmania University. He is working as an associate professor and head in Department of Mathematics at JNTUH College of Engineering, Jagitial. He has 18 years of research and Engineering Teaching experience. In his long tenure of service, he worked in various a dministration positions. His area of the research is Magneto-hydrodynamics fluid flows. To his credit, he has guided two M. Phil. Degree and submitted a Thesis for $\mathrm{Ph}$.D. degree. Presently, he is guiding $2 \mathrm{M}$. Phil and 5 Ph.D. candidates. He has published several papers in reputed National and International Journals and he is also a life member of reputed several professional societies. 